\title{
In Vitro Evaluation of Enzymatic and Antifungal Activities of Soil- Actinomycetes Isolates and Their Molecular Identification by PCR
}

\author{
Nasser Keikha ${ }^{1}$; Seyyed Amin Ayatollahi Mousavi ${ }^{2, *}$;Ali Reza Nakhaei ${ }^{3}$; Mohammad Hossein \\ Yadegari ${ }^{4}$; Gholam Hossein Shahidi Bonjar ${ }^{5}$; Somayyeh Amiri ${ }^{6}$ \\ ${ }_{2}^{1}$ Department of Laboratory Sciences, Faculty of Medicine, Zahedan University of Medical Sciences, Zahedan, IR Iran \\ ${ }_{3}$ Department of Medical Mycology and Parasitology, Faculty of Medicine, Kerman University of Medical Sciences, Kerman, IR Iran \\ ${ }_{4}^{3}$ Department of Biochemistry, Faculty of Medicine, Zahedan University of Medical Sciences, Zahedan, IR Iran \\ ${ }_{5}^{4}$ Department of Medical Mycology, Faculty of Medicine, Tarbiat Modares University, Tehran, IR Iran \\ ${ }_{6}^{5}$ Department of Plant Pathology, Faculty of Agricultural Engineering, Shahid Bahonar University of Kerman, Kerman, IR Iran \\ Zahedan Economic and Finance Organization, Zahedan, IR Iran \\ ${ }^{*}$ Corresponding author: Seyyed Amin Ayatollahi Mousavi, Department of Medical Mycology and Parasitology, Faculty of Medicine, Kerman University of Medical Sciences, Kerman, \\ IR Iran. Tel: +98-9133418009, E-mail: aminayatollahi@yahoo.com.
}

Received: September 16, 2013; Revised: May 28, 2014; Accepted: July 4, 2014

\begin{abstract}
Background: Human cutaneous infection caused by a homogeneous group of keratinophilic fungi called dermatophytes. These fungi are the most common infectious agents in humans that are free of any population and geographic area. Microsporum canis is a cause of dermatophytosis (Tinea) in recent years in Iran and atypical strain has been isolated in Iran. Its cases occur sporadically due to $M$. canis transmission from puppies and cats to humans. Since this pathogenic dermatophyte is eukaryotes, chemical treatment with antifungal drugs may also affect host tissue cells.

Objectives: The aim of the current study was to find a new antifungal agent of soil-Actinomycetes from Kerman province against M. canis and Actinomycete isolates were identified by PCR.

Materials and Methods: A number of hundred Actinomycete isolated strains were evaluated from soil of Kerman province, for their antagonistic activity against the M. canis. M. canis of the Persian Type Culture Collection (PTCC) was obtained from the Iranian Research Organization for Science and Technology (IROST). Electron microscope studies of these isolates were performed based on the physiological properties of these antagonists including lipase, amylase, protease and chitinase activities according to the relevant protocols and were identified using gene16SrDNA.

Results: In this study the most antagonist of Actinomycete isolates with antifungal activity against M. canis isolates of L1, D5, Ks1m, Km2, Kn1, Ks8 and Ks1 were shown in vitro. Electron microscopic studies showed that some fungal strains form spores, mycelia and spore chain. Nucleotide analysis showed that Ks8 had maximum homology (98\%) to Streptomyces zaomyceticus strain xsd08149 and L1 displayed 100\% homology to Streptomyces sp. HVG6 using 16SrDNA studies.

Conclusions: Our findings showed that Streptomyces has antifungal effects against M. canis.
\end{abstract}

Keywords: Actinomycetes; Microsporum canis; Antifungal; 16SrDNA

\section{Background}

Human cutaneous infections caused by a homogeneous group of keratinophilic fungi called dermatophytes. These ubiquitous fungi are responsible for the most common fungal infections in major populations globally (1). Microsporum canis is the main agent of dermatophytosis in cats, its natural host and is responsible for a frequent zoophilic that has increased in several European countries $(2,3)$. M. canis is the major common cause of baldness in recent years in Iran. There also are reports of pseudomycetoma by $M$. canis. $M$. canis is a zoonosis fungus in dogs, cats, probably horses and monkeys that can lead to infection. While it is not geophilic but the isolates from soil cause ectothrix form of tinea capitis in human. In case of infection due to $\mathrm{M}$. $\mathrm{ca}$ - nis, hairs show greenish-yellow to brilliant fluorescence radiation if tested with Wood's lamp. This fungus also attacks to the skin with no hair $(4,5)$. Identify M. canis virulence factors have focused on proteases, including keratinases (6). This pathogenic dermatophyte is eukaryote and chemical treatment with antifungal drugs may also affect host tissue cells (7).

Many species of Actinomycetes, particularly those belonging to the genus Streptomyces, are well known as antifungal biocontrol agents that inhibit several plant pathogenic fungi $(8,9)$. The antagonistic activity of Streptomyces to fungal pathogens is usually related to the production of antifungal compounds $(10,11)$ and extracellular hydrolytic enzymes $(11,12)$. Chitinase and $\beta$-1, 3-glucanase are

Copyright (C) 2015, Ahvaz Jundishapur University of Medical Sciences. This is an open-access article distributed under the terms of the Creative Commons Attribution-NonCommercial 4.0 International License (http://creativecommons.org/licenses/by-nc/4.0/) which permits copy and redistribute the material just in noncommercial usages, provided the original work is properly cited. 
considered to be important hydrolytic enzymes in the lysis of fungal cell walls, as for example, cell walls of $\mathrm{Fu}$ sarium oxysporum, Sclerotinia minor, and S. rolfsii (13). The antifungal potential of extracellular metabolites from Streptomyces against some fungi was previously reported $(8,10)$. However, data related to the antagonistic ability of the extracellular metabolites of Streptomyces strains to suppress the growth of the fungal pathogens Colletotrichum gloeosporioides and S. rolfsii having a broad host range are limited (9).

\section{Objectives}

This study aims to determine the effect of soil Actinomycetes antifungal activity against $M$. canis isolated from soil and identification like Streptomyces DNA using gene 16SrDNA.

\section{Materials and Methods}

\subsection{Sampling, Isolation and Culture of Actinomy- cetes}

To obtain Actinomycete isolates over 100 random soil samples from different areas of Kerman Province (Sangi Park, Sahebazzaman the mosque, Motahari Park and Neshat Park) from depth of $10-20 \mathrm{~cm}$ of the ground were taken, air dried and passed through $0.8 \mathrm{~mm}$ mesh. Tenfold serial dilutions prepared in water $(10-4,10-5$ and 10 - 6) then cultured on Casein Glycerol Agar (CGA) (casein $0.3 \mathrm{~g} / \mathrm{L} ; \mathrm{KNO}_{3} 2.0 \mathrm{~g} / \mathrm{L} ; \mathrm{NaCl} 2.0 \mathrm{~g} / \mathrm{L} ; \mathrm{MgSO}_{4} .7 \mathrm{H}_{2} \mathrm{O} 0.05 \mathrm{~g} / \mathrm{L}$ $\mathrm{CaCO}_{3} 0.02 \mathrm{~g} / \mathrm{L} ; \mathrm{FeSO}_{4} \cdot \mathrm{H}_{2} \mathrm{O} 0.01 \mathrm{~g} / \mathrm{L} ; \mathrm{KH}_{2} \mathrm{PO}_{4} 2.0 \mathrm{~g} / \mathrm{L}$; and agar (Merck, Germany) $18.0 \mathrm{~g} / \mathrm{L}$ ) and incubated at $29^{\circ} \mathrm{C}$ for 5-7 days. From day five on, Actinomycete colonies were isolated in pure cultures on CGA. Full grown cultures kept refrigerated before use.

\subsection{In Vitro Bioassays to Detect the Antifungal Ac- tivities}

Bioassays were performed using Disk-method technique. In this method, six mm agar plugs of six-day old cultures of Actinomycetes on CGA medium were placed on lawn cultures of the pathogen on Potato Dextrose Agar (PDA) (Merck, Germany). Plates incubated at $28^{\circ} \mathrm{C}$ for 10 days. Actinomycetes with clear inhibition zones were recorded as positive and selected for further evaluations. Plain agar plugs were used as controls. All bioassays were performed in triplicates and the mean values calculated.

\subsection{Determination of Minimum Inhibitory Concen- tration (MIC)}

To determine the MIC, concentrations of crude sap of each antagonist was prepared as 10, 5, 2.5, 1.25, and 0.625 $\mathrm{mg} / \mathrm{mL}$ in DMSO: MeOH (Merck, Germany) (1:1, v/v) with suspension of $M$. canis conidia and tested in well-method technique against the pathogen and incubated at $29^{\circ} \mathrm{C}$ for 12 days. The lowest concentration with growth inhibition was selected as MIC. All bioassays performed in triplicates and the mean values calculated.

\subsection{Production of Extracellular Enzymes}

Lipase activity test: Growth media containing $10 \mathrm{~g}$ peptone (Merck, Germany), $5 \mathrm{~g} \mathrm{NaCl}$ and $15 \mathrm{~g}$ agar/L was prepared and autoclaved. After autoclaving, $10 \mathrm{mg}$ of Tween 80 was added to the media and mixed gently. Disk plugs $(6 \mathrm{~mm})$ of six-day old cultures of antagonist were prepared and placed on the media as in disk-method bioassay. Hydrolysis of Tween 80 by the bacterium presented as distinctive zone around the bacterial plug (14).

\subsection{Proteolytic Activity Test}

Minimal medium containing one g of sucrose (Merck, Germany), three $\mathrm{g}$ of casein (Merck, Germany), $2 \mathrm{~g}$ of calcium chloride and $15 \mathrm{~g}$ of agar/L in distilled water was prepared and autoclaved. $6 \mathrm{~mm}$ antagonist disk plugs were prepared and placed on the media as in disk-method bioassay. Hydrolysis of casein and production of clear zones were evaluated positive (15).

\subsection{Amylase Activity Test}

To $5 \%$ water agar, $2 \%(\mathrm{w} / \mathrm{w})$ starch was added, autoclaved and poured in Petri dishes. After cooling, $6 \mathrm{~mm}$ antagonist disk plugs were prepared and placed on the media as in disk-method bioassay. Hydrolysis of starch and production of clear zones were evaluated positive (16).

\subsection{Chitinase Activity Test}

Nutrient agar was supplemented with $4 \%(\mathrm{w} / \mathrm{w})$ colloidal chitin (Sigma), autoclaved and poured in Petri dishes. After cooling, $6 \mathrm{~mm}$ antagonist disk plugs were prepared and placed on the media as in disk-method bioassay. Hydrolysis of colloidal chitin and production of clear zones were evaluated positive. In this media, chitin was used as the sole carbon source and, therefore, strains with chitinase activity, hydrolysed chitin and transparent halo around their colonies were evaluated positive and Electron microscope studies of active isolates were performed according to the relevant protocol (17).

\subsection{Genetic Analysis of PCR}

Extraction of bacterial DNA: From 5 day old bacterial culture, $1.5 \mathrm{~mL}$ transferred to sterile microtube and centrifuged for 5 minutes at $7500 \mathrm{~g}$ till bacteria precipitated. The supernatant discarded, the pellet received $100 \mu \mathrm{L}$ protease buffer and kept at $95^{\circ} \mathrm{C}$ for 10 minutes solution was added to sediment bacteria according to the kit of Bacterial DNA extraction Owned by Sina Colon Iran. The lysis solution was given a gentle shake for 10 minutes at $37^{\circ} \mathrm{C} .100 \mu \mathrm{L}$ of the sample with $400 \mu \mathrm{L}$ lysis solution were mixed and shake for 15 - 20 seconds to be rotated. 
Keikha Netal.

Then $300 \mu \mathrm{L}$ of alcohol was added to microtube for 20 minutes at $-20^{\circ} \mathrm{C}$. The microtube was centrifuged for 10 minutes at $12000 \mathrm{~g}$. The supernatant was discarded and the microtube gently was stroke on a paper for 2-3 seconds to remove the remaining precipitated liquid. One $\mathrm{mL}$ of wash buffer added to microtube for 3 - 5 seconds and slowly moved into rotator. It was then centrifuged at $12000 \mathrm{~g}$ for 5 minutes and the supernatant was removed. The microtube was kept to dry for 5 minutes at $65^{\circ} \mathrm{C}$. After PCR, $45 \mu \mathrm{L}$ of the PCR products including of the forward primer and molecular identification based on 16SrDNA was sent to the Bioneer Company for sequencing (18). Molecular identification: Sequences were identified using BLAST from National center for Biotechnology Gene Sequences in the database (http:www.ncbi. nlm.gov/BLAST/). This molecular method for identification of sequenced isolates using the 16SrDNA was found at the species level.

\section{Results}

\subsection{Actinomycetes Isolated From Soil}

From $10^{-4}-10^{-6}$ dilutions of soil samples, over 100 Actinomycetes isolated in pure cultures on which further investigations performed. In vitro antifungal activity of Actinomycete isolates: Bioassay result of Actinomycete isolates Ks8 and L1 against the tested dermatophyte evaluated in disk-method technique. The inhibition zones in which no visible growth of the pathogen observed are representative of antifungal activity.

\subsubsection{Determination of Minimum Inhibitory Concentra- tion (MIC)}

As shown in the Figure 1, The MIC for both Actinomycete isolates of L1 and Ks8 isolates determined as $25 \mathrm{mg} / \mathrm{mL}$.

\subsection{Enzymatic Bioassays}

\subsubsection{Lipase Activity}

L1 isolate had varying degrees of lipase activity and could hydrolyze tweens. Sedimentary halo formation around colonies was produced by the L1 isolate of Actinomycete.

\subsubsection{Proteolytic Activity}

Extracellular protease activity of Ks8 and L1 Actinomycete isolates was indicated by their ability to hydrolyze casein (production of clear halo around colonies).

\subsubsection{Amylase Activity}

L1 and Ks8 isolates was able to break down the starch polymer and created the colorless zone around the colonies after adding the substrate as shown in the Figure 2.

\subsubsection{Chitinase Activity}

L1 and Ks8 isolates had the best result for chitinase activity. It showed a halo around the colony after growing on the chitin media.

\subsubsection{Electron Microscope Studies}

Electro micrographs of both Actinomycete isolates of Ks8 and L1 indicated that both isolates bear long spore chains in which spores had smooth surfaces. Figure 3 shows the spore chains and mycelial morphology of both isolates. Phylogenetic analysis of the nucleotide sequence of the 16SrDNA gene of active Actinomycete isolates: 16SrDNA sequences using specific primers was amplified by PCR technique (Figures 4 and 5). Comparison of 16SrDNA sequences active Actinomycete isolates with sequences available in Gen Bank/DDBJ/EMBL using Blast. Nucleotide analysis showed that isolate Ks8 had maximum homology (98\%) to Streptomyces zaomyceticus strain xsdo8149 and L1 displayed 100\% homology to Streptomyces sp. HVG6 (Figure 6).

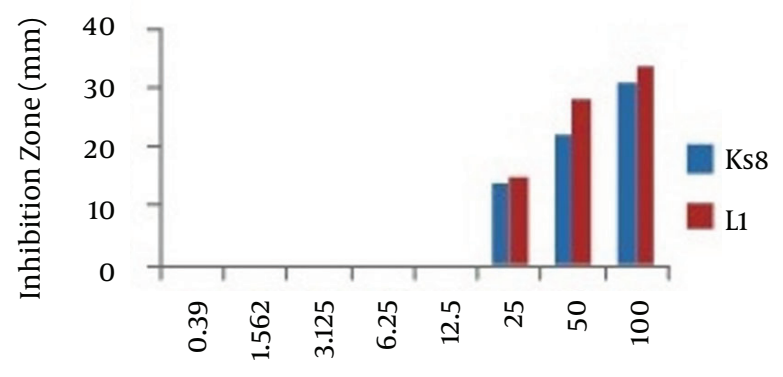

Concentration $(\mathrm{mg} / \mathrm{ml})$

Figure 1. Minimum Inhibitory Concentration (MIC) of Crude Extracts of Actinomycete Isolates Ks8 and L1 Against the Tested Dermatophyte Evaluated in well-Method Technique.

Figure 2. Amylase Activity

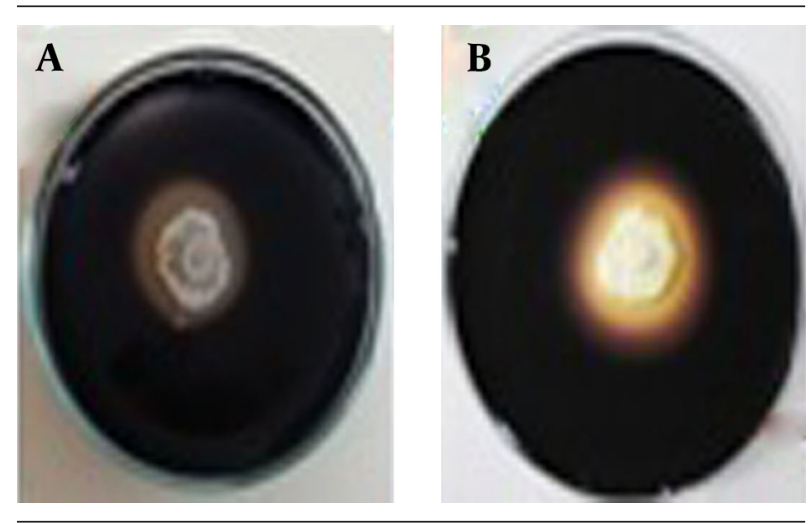

A) L1 Actinomycete isolate, B) Ks8 Actinomycete isolate. 

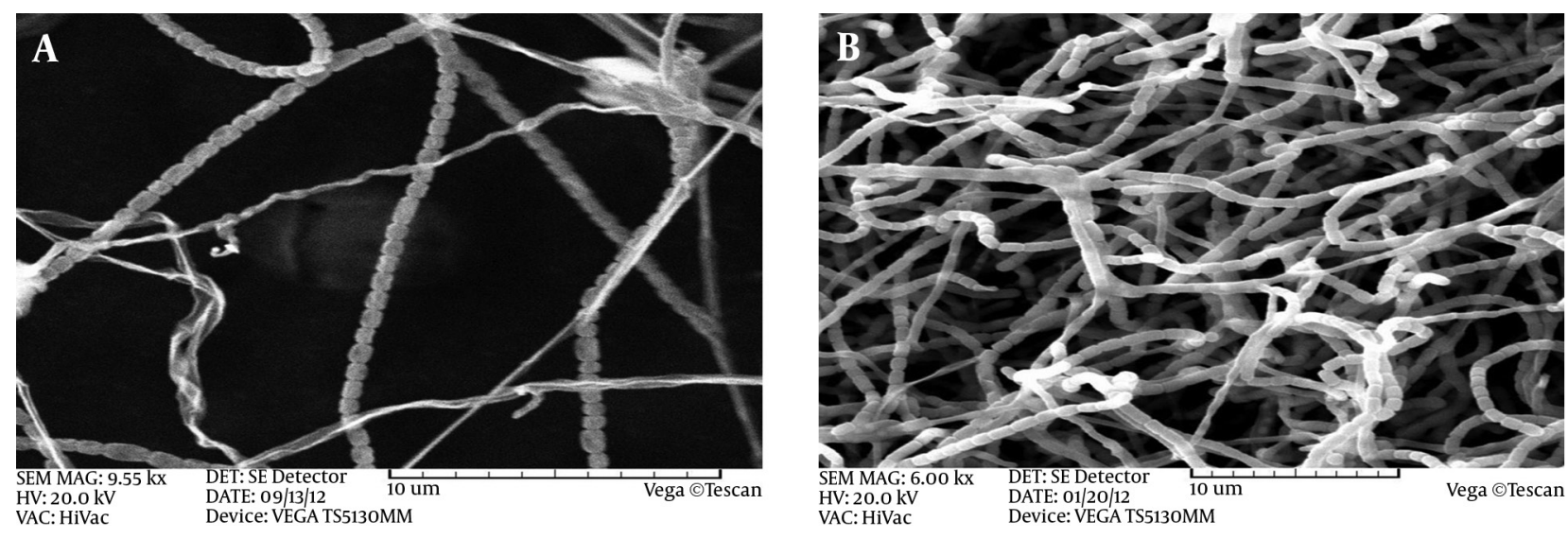

Figure 3. Electromicrographs of Actinomycete Isolates Ks8 (A) and L1 (B). Both isolates bear long spore chains in which spores have smooth surfaces.

A

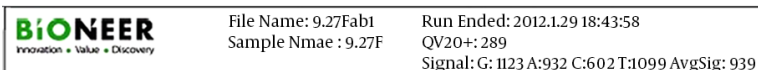

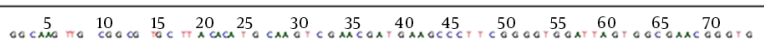

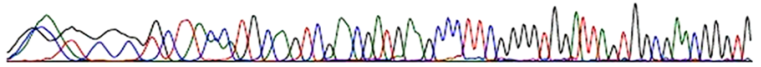

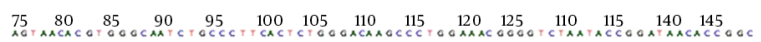

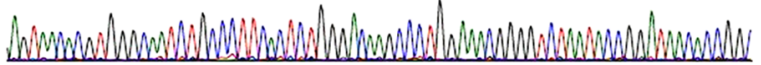

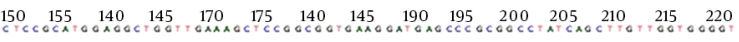

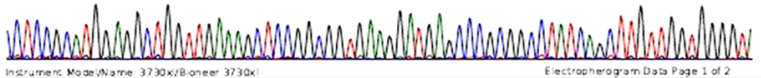

B

\begin{tabular}{lll|}
\hline BíONEER & File Name:10.27Fab1 & Run Ended:2012.1.29 18:43:58 \\
morson. & Sample Nmae:10.27F & QV20+: 284 \\
& & Signal:G: 1718 A:1477 C:1021 T:1922 AvgSig:1534
\end{tabular}

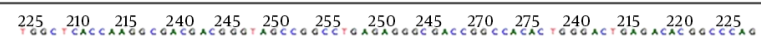

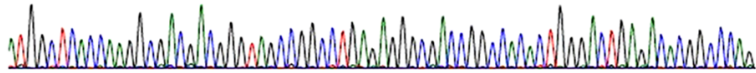

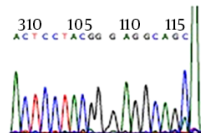

Figure 4. Histogram of Ks8Sequence Active Actinomycete Isolate

A

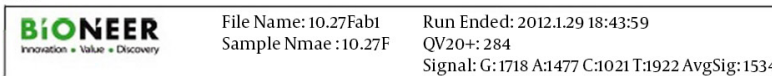

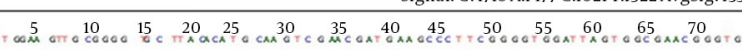

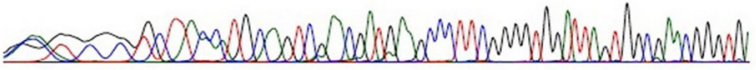

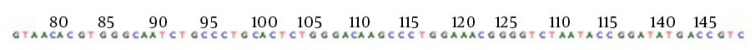

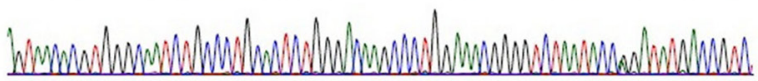

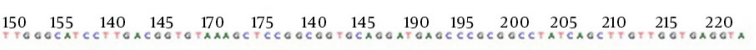

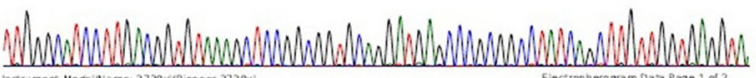

Figure 5. Histogram of L1 Sequence Active Actinomycete Isolate
B

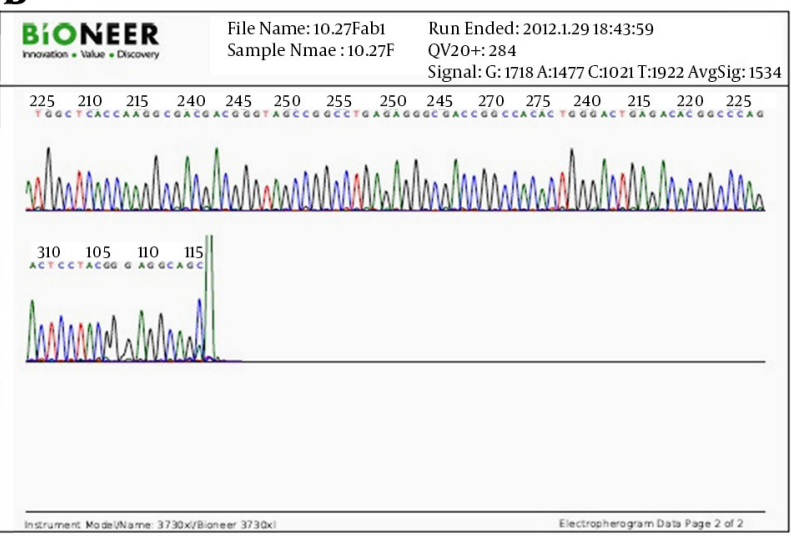


Keikha Netal.

A

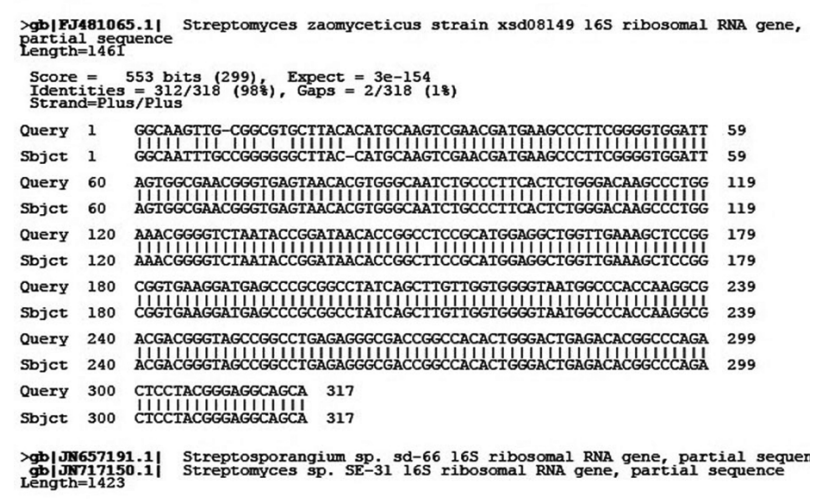

\section{B}

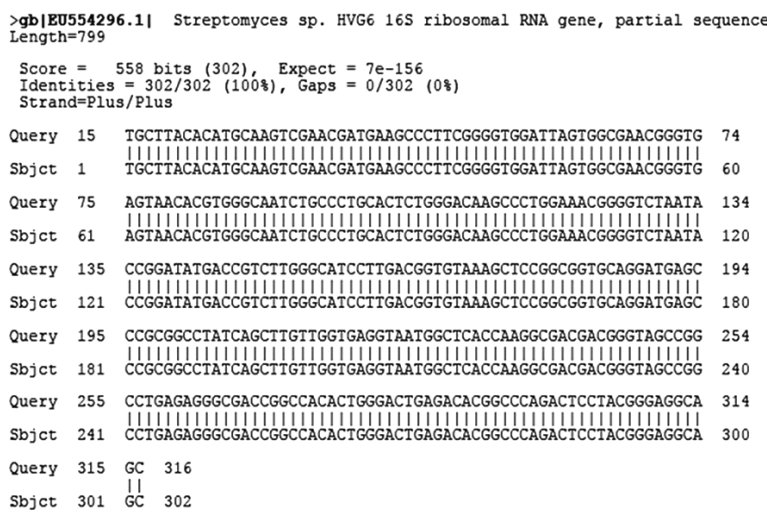

Figure 6. A): Sequence Alignment of Streptomyces Zaomyceticus Strain xsdo8149 in the NCBI Database Sequences With Ks8Sequence Active Actinomycete Isolate. B): Sequence Alignment of Streptomyces sp. HVG6 in the NCBI Database Sequences With L1 Sequence Active Actinomycete Isolate.

\section{Discussion}

It has been demonstrated that 85 percent of antibiotics which produced and used from Actinomycetes are mainly from various species of Streptomyces (19). In this study, we tried to screen and evaluate Actinomycetes isolated from the soil of Kerman Province for their antagonist activity against $M$. canis the causative agent of human dermatophytosis. Over 100 isolate of Actinomycetes cultures were isolated from different areas of Kerman's soil and then the in vitro tests were performed. Actinomycetes were isolated when their morphological features and their pigmentation revealed after seven days (20). Lakshmipathy and Kannabiran (2009) introduced 100 isolates of Actinomycetes isolated from soil (21). In an initial screening to find appropriate antifungal effect of isolates against M. canis, biological screenings performed by agar disk method. The isolates of L1, D5, Ks10, Km2, Kn10, Ks8 and Ks1 showed highest antagonist activity against $M$. canis based on production of zones of inhibition. In a similar study by Shahidi Bonjar, et al. (2006) they identified twelve strains out of 130 isolates of Actinomycetes against Phytophthoradrexler (22).

In another study in Bangkok (2008) similar to what we did; 10 Actinomycetes isolated from 146 soil samples showed antifungal effect against the three pathogenic fungi (23). Augustine and colleagues (2005) also conducted a similar study that proved Streptomyces rocky AK39 has anti-dermatophyte effect (24). Based on their MIC studies they demonstrated only three isolates from more than one hundred strains showed to have antifungal compounds against the tested fungi. In this research antifungal activity of the isolated strains was demonstrated and it showed the importance and potential for further investigation. Lateral diffusion is a physicochemical approach in which microorganisms using it as indicators of active compounds (25). The study conducted by Zakir and colleagues (2002) developed a MIC between 32 - 64 mg/mL for the active metabolite isolated from Streptomyces species against four Gram positive and Gram-negative bacteria respectively (26). As isolates evaluated in this study able to control fungi at a very low concentration then it can be concluded that the antagonistic effects are well studied in the control of microorganisms.

The SEM images obtained from isolates of Actinomycetes showed some form spores, mycelia and spore chains as their morphology revealed. Research in order to identify and classify different species of Streptomyces and electrontype levels of spores in Streptomyces species were reported straight. Also the form of spore chains using electron microscopic studies of Streptomyces species, and Streptomyces griseus was a spiral, circular and flexible straight up (27). Streptomyces lytic activities are mainly the result of lyse enzymes such as chitinase and gluconase (28). Chitin is a major component of the fungal cell wall and as substrate for chitinase enzyme (29).

The inhibition of fungi by Streptomyces may be related to the production of chitinase (30). In a study by Baharlouei et al. (2009) they reported 18 Actinomycete strains out of 110 isolates had strong chitinase activity (31). Since the fungal cell wall contains chitin fibers and the matrix of proteins proteases play a significant role in the degradation of the wall (32). The role of extracellular proteases in various biocontrol processes also was shown in pathogenic fungus Trichoderma harzianum (33). In this research we demonstrated that Streptomycetes antagonists had antifungal effects over M. canis. The outcome of this study can be used for making new antifungal drugs in the future. In this case many various elements should be studied such as evaluation on animal's models for short and long term side effects, carcinogenic and teratogenic effects and environmental impacts. The topical evaluations on volunteers also should be considered before any real conclusion. 


\section{Acknowledgements}

We thank the Kerman University of Medical Sciences for the financial supports.

\section{Funding/Support}

This study was part of a M.Sc thesis supported by Deputy of Research University of Medical Sciences (grant 90/10/60/30963).

\section{References}

1. Weitzman I, Summerbell RC. The dermatophytes. Clin Microbiol Rev. 1995;8(2):240-59.

2. Scott DW, Miller WH, Griffin CE. Fungal Skin Diseases In Small Animal Dermatology. 5th edPhiladelphia: PA; 1995.

3. Lunder M, Lunder M. Is Microsporum canis infection about to become a serious dermatological problem? Dermatology. 1992;184(2):87-9.

4. Zaini F, Mahbod A, Emami M. Omprehencive Medical Mycology, Hand Book. 4TH ed, Tehran: Tehran UP; 2011.

5. Larone DH. Medically Important Fungi - A Guide to Identification. 3rd edWashington: ASM Press; 1995.

6. Descamps F, Brouta F, Vermout S, Monod M, Losson B, Mignon B Recombinant expression and antigenic properties of a $31.5-\mathrm{kDa}$ keratinolytic subtilisin-like serine protease from Microsporum canis. FEMS Immunol Med Microbiol. 2003;38(1):29-34

7. Klepser ME, Ernst EJ, Pfaller MA. Update on antifungal resistance. Trends Microbiol. 1997;5(9):372-5

8. Joo GJ. Production of an anti-fungal substance for biological control of Phytophthora capsici causing phytophthora blight in red-peppers by Streptomyces halstedii. Biotechnol Lett. 2005;27(3):201-5.

9. Errakhi R, Bouteau F, Lebrihi A, Barakate M. Evidences of Biological Control Capacities of Streptomyces Spp. Against Sclerotium Rolfsii Responsible for Damping-off Disease in Sugar Beet (Beta vulgaris L.). World J Microb Biot. 2007;23(11):1053-9.

10. Fourati-Ben Fguira L, Fotso S, Ben Ameur-Mehdi R, Mellouli L, Laatsch $\mathrm{H}$. Purification and structure elucidation of antifungal and antibacterial activities of newly isolated Streptomyces sp. strain US80. Res Microbiol. 2005;156(3):341-7.

11. Trejo-Estrada SR, Paszczynski A, Crawford DL. Antibiotics and enzymes produced by the biocontrol agent Streptomyces violaceusniger YCED-9. J Ind Microbiol Biot. 1998;21(1-2):81-90.

12. Mukherjee G, Sen SK. Purification, characterization, and antifungal activity of chitinase from Streptomyces venezuelae P10. Curr Microbiol. 2006;53(4):265-9.

13. El-Tarabily KA, Soliman MH, Nassar AH, Al-Hassani HA, Sivasithamparam K, McKenna F, et al. Biological Control of Sclerotinia Minor Using a Chitinolytic Bacterium and Actinomycetes. Plant Pathol.2000;49(5):573-83.

14. Sierra G. A simple method for the detection of lipolytic activity of micro-organisms and some observations on the influence of the contact between cells and fatty substrates. Antonie Van Leeuwenhoek. 1957;23(1):15-22.

15. Dunne C, Moenne-Loccoz Y, de Bruijn FJ, O'Gara F. Overproduction of an inducible extracellular serine protease improves biological control of Pythium ultimum by Stenotrophomonas maltophilia strain W81. Microbiology. 2000;146 ( Pt 8):2069-78.
16. Pelczar MJ, Chairman J, Bard RC, Burnett GW, Conn HJ, Demoss RD, et al. Manual of Microbiological Methods.McGraw-Hill, New York; 1951.

17. Hsu SC, Lockwood JL. Powdered chitin agar as a selective medium for enumeration of actinomycetes in water and soil. Appl Microbiol. 1975;29(3):422-6.

18. Cook AE, Meyers PR. Rapid identification of filamentous actinomycetes to the genus level using genus-specific 16S rRNA gene restriction fragment patterns. Int J Syst Evol Microbiol. 2003;53(Pt 6):1907-15.

19. Walsh TJ. Management of immunocompromised patients with evidence of an invasive mycosis. Hematol Oncol Clin North Am. 1993;7(5):1003-26.

20. Moosavi SMA, Ghanbarvand F, Dehnad A. Growth inhibitory and differentiating effects of ethyl acetate soluble metabolite of Iranian native bacteria, Streptomyces calvus, in human myeloid leukemia K562 cell line. Med SCi J Islamic Azad Univ. 2012;22(3):175-83.

21. Lakshmipathy DT, Kannabiran K. A Morphological, Biochemica and Biological Studies of Halophilic Streptomyces sp. Isolated from Saltpan Environment. Am. J. Infect. 2009;5(3):200-6.

22. Shahidi Bonjar GH, Barkhordar B, Pakgohar N, Aghighi S, Biglary S, Rashid Farrokhi P, et al. Biological Control of Phytophthora Drechsleri Tuker the Causal Agent of Pistachio Gummosis Under Greenhouse Conditions by Use of Actinomycetes Plant Pathol J. 2006;5(1):20-3.

23. Prapagdee B, Kuekulvong C, Mongkolsuk S. Antifungal potential of extracellular metabolites produced by Streptomyces hygroscopicus against phytopathogenic fungi. Int J Biol Sci. 2008;4(5):330-7.

24. Augustine SK, Bhavsar SP, Kapadnis BP. Production of a growth dependent metabolite active against dermatophytes by Streptomyces rochei AK39. Indian J Med Res. 2005;121(3):164-70.

25. Hewitt W. Theory and Application of Microbiological Assay.1st edSan Diego: Academic Press; 1998.

26. Zakir Sultan M, Ara Khatune N, Sultana Sathi Z, Shah Alam Bhuiyan MD, Golam Sadik M, Akteruzzaman Choudury M, et al. In Vitro Antibacterial Activity of an Active Metabolite Isolated from Streptomyces Species. Biotechnol J. 2002;1(2 SPPL 4):100-7.

27. Locci R. In: Streptomycetes and related genera. Eds: Williams ST, Sharpe ME and Holt JG)., editor. Vol. 4. USA: Williams \& Willkins; 1989. pp. 2451-2492.

28. Ito M, Maruyama T, Saito N, Koganei S, Yamamoto K, Matsumoto N. Killer cell lectin-like receptor G1 binds three members of the classical cadherin family to inhibit NKcell cytotoxicity.J Exp Med 2006;203(2):289-95.

29. Hoell IA, Dalhus B, Heggset EB, Aspmo SI, Eijsink VG. Crystal structure and enzymatic properties of a bacterial family 19 chitinase reveal differences from plant enzymes. FEBS J. 2006 273(21):4889-900.

30. Gohel V, Singh A, Vimal M, Ashwini P, Chhatpar HS. Bioprospecting and Antifungal Potential of Chitinolytic Microorganisms. Afr J Biotechnol. 2006(5):54-72.

31. Baharlouei A, Sharifi Sirchi GR, Shahidi Bonjar GH. [Isolation, Cloning and Sequencing of Chitinase Gene from Streptomyces Plicatus]. Natl Biotechnol Congr Iran. 2009.

32. Flores JM, Castillo VB, Franco FC, Huata AB. Superficial fungal in fections: clinical and epidemiological study in adolescents from marginal districts of Lima and Callao, Peru. J Infect Dev Ctries. 2009;3(4):313-7.

33. Sivan A, Chet I. Degradation of Fungal Cell Walls by Lytic Enzymes of Trichoderma Harzianum. Gen Microbiol J.1989(135):675-82. 